\title{
Research and Application of Lithium Iron Phosphate Battery in Substation BMS
}

\author{
Yin yuheng $^{1 *}$ and Wang lei ${ }^{2}$ \\ ${ }^{1}$ School of automation, Harbin University of Science and Technology, \\ Heilongjiang Harbin 150080, China \\ ${ }^{2}$ Electric Power Research Institute of Heilongjiang Province ,Heilongjiang \\ Harbin 150090,China \\ 13503636310@163.com
}

\begin{abstract}
With the development and application of lithium iron phosphate battery, more and
\end{abstract} more power battery in DC system using lithium iron phosphate batteries to replace original lead-acid battery. The lithium iron phosphate battery management system (BMS) is the focus of this paper, the communication between the battery management system and the substation monitor of DC system, effectively ensure the battery and the DC system is safe and reliable to work. How to grasp the variation rule of the internal state of the battery and the influence of external factor on the capacity of the battery, to establish a reasonable and effective battery model Gand algorithrof SOC and realize SOC estimation and reduce the estimation error, which is the most basic and the most important aspect of the battery safety management.

Keywords: DC system; lithium iron phosphate batteries; SOC

\section{Introduction}

In the current substation, modern EHV large power projects, large units, and modern city network construction project, the quality of the DC system has an important impact on the safe operation of powet grid. The stability of DC system of the normal operation of the transformer substation plays a key role, and also guarantee the safe operation of the transformer substation. Because the battery is the key component of DC system, the core work of DC system is scientific maintaining battery, the battery charge and discharge management is reasonable to ensure the long time operation.

In many chemical batteries, lead-acid batteries is currently widely used in all kinds of substation, but for the reason of lead pollution, some lead-acid battery production units have been ordered to shut down. This is bound to cause rise in lead-acid battery prices, the use rate of decline. Because the nickel metal hydride battery and nickel cadmium battery have large volume, short life cycle and memory efficiency, the application is restricted. In recent years, the lithium ion battery has obtained considerable development, become an important member of the family of battery. Lithium ion battery is high capacity rechargeable battery developed in the 1990s, and has the advantages of is able to store more energy, energy ratio, cycle life, self-discharge rate, no memory effect and no environmental pollution. Cathode materials for lithium ion batteries is lithium cobalt oxide, lithium manganese oxide and lithium iron phosphate, the cathode is carbon material. Because high prices and heavy pollution of the raw materials of cobalt and manganese, the performance of active and explosive, current research and application of battery industry are mainly concentrate on the low cost lithium iron phosphate battery. Lithium iron phosphate batteries have the advantage of stable structure, good circulation performance, excellent safety, that other battery do not have, which makes it has very 
broad market prospects. Therefore, the lithium iron phosphate battery can be used as a battery DC system of substation.

The lithium iron phosphate battery management system (BMS) is the focus of this paper, the communication between the battery management system and the substation monitor of DC system, effectively ensure the battery and the DC system is safe and reliable to work. How to grasp the variation rule of the internal state of the battery and the influence of external factors on the capacity of the battery, to establish a reasonable and effective battery model and algorithm of SOC and realize SOC estimation and reduce the estimation error, which is the most basic and the most important aspect of the battery safety management. Safety management mainly refers to the safety of working condition of the battery, including excessive charge and excessive discharge control, overcurrent protection, high-voltage isolation, balanced processing and thermal management. When the battery is abnormal the BMS should immediately start the protection function of the battery controp module, ensure the battery can run in the normal way.

\section{The Introduction of Lithium Iron PhosphateBattery}

\subsection{The Structure of Lithium Iron Phosphate Battery}

The internal structure of lithium iron phosphate batteryas shown in Figure 1. On the left is olivine type lithium iron phosphate as the positive electrode of the battery, connected by the aluminum foil with the positive electrode of the battery, in the middle is diaphragm of the polymer, separates the positive electrode and the negative electrode of a battery, lithium ion pass but electrons cannot, and on the right is composed of carbon or graphite cathode, connected the copper foil and the battery cathode. The internal battery is filled with electrolyte, the external is encapsulated by metal shell.

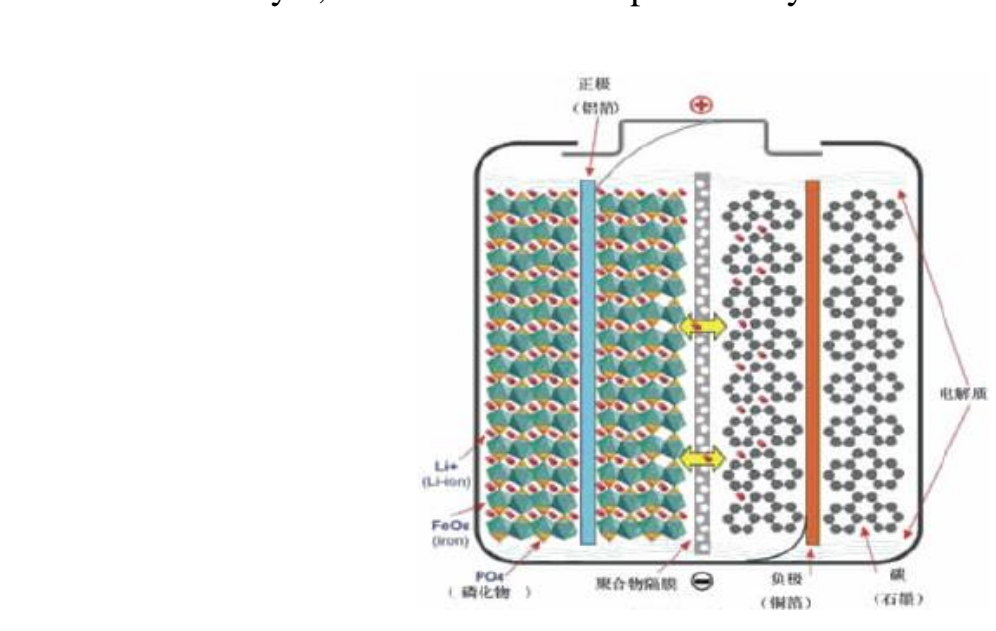

Figure 1. The Internal Structure of Lithium Iron Phosphate

\section{2. Lithium Iron Phosphate Battery Advantage}

(1) Long life. The cycle life of long life lead-acid battery in about 300 times, the maximum is 500, while the lithium iron phosphate battery, cycle life is more than 2000 times, when the standard charge (5 hour rate), can reach 2000 times. At the same quality, lead-acid battery is "half a year of the new, half a year of the old, and half a year of the maintenance", in the application of the electric power industry at most 5-8 years, while the lithium iron phosphate battery under the same conditions will reach 8-10 years.

(2) The safety use. Lithium iron phosphate completely solve the problem of the potential safety hazard of $\mathrm{LiCoO} 2$ and $\mathrm{LiMn} 2 \mathrm{O} 4, \mathrm{LiCoO} 2$ and $\mathrm{LiMn} 2 \mathrm{O} 4$ under the strong 
collision would produce an explosion, pose a threat to the safety of electric power, and lithium iron phosphate through rigorous safety testing, even in the worst traffic accident would not have exploded.

(3) Large current fast charging and discharging. The lithium iron phosphate battery large current $(2 \mathrm{C}$ current) fast charging and discharging, in special charger, charging current of $1.5 \mathrm{C}$ within 40 min can make the battery is full, the starting current is up to $2 \mathrm{C}$, and now the lead-acid battery does not have this performance.

(4) Thermostability. Lithium iron phosphate electric heating peak is up to $350{ }^{\circ} \mathrm{C}-500$ ${ }^{\circ} \mathrm{C}$ and lithium manganese oxide and lithium cobalt oxide in only $200{ }^{\circ} \mathrm{C}$. A wide range of working temperature $\left(-20{ }^{\circ} \mathrm{C}-75^{\circ} \mathrm{C}\right)$, high temperature resistant properties.

(5) No memory effect. Rechargeable batteries are often not put in full under the working conditions, the capacity will rapidly lower than the rated capaefity, this phenomenon called memory effect. As the nickel metal hydride battery, nickel cadnum battery exist memory effect, but lithium iron phosphate battery without this phenomenon, no matter what is the status of the battery, can be charged with use, without use out after recharging.

(6) The green environmental protection. The batterydoes not contain any heavy metals and rare metals (nickel metal hydride batteries need rare metals), non-toxic (Passed the SGS certification), no pollution, in line with the European RoHS requirements for absolute green batteries. There are a large number of lead in the lead-acid batteries, in the waste if not handled properly, will cause secondary polution to the environment, and lithium iron phosphate materials both in production and use, no pollution.

\section{The Design of Battery Management System}

The substation battery is composed of a plurality of single batteries connected in series. In the case of using the battery directly without equip battery management system, due to the differences between monlomers in the battery group in the use of the process will gradually increase, and cannot be adjusted, then lead to the battery after running for a period of time appear monomer dvercharge, over discharge, over current and battery temperature is too high, a serres of dault, resulting in reduced service life of the battery, performance degradation, serious waste, explosion and so on. Therefore, it is very necessary for lithium irøn phosphate battery to equip with a battery management system. The battery managemen system can solve the security problem of battery effectively, so as to ensure the reliable operation of the battery, and prolong the service life of the battery, reduce battery costs.

According to the actual needs of the lithium iron phosphate battery management system, battery management system adopts distributed structure, the battery management system (BMS) and the temperature acquisition unit (BVT). Under the total control of batte management system (BMS), respectively, to achieve the various functions required for battery management system using a plurality of control units, such as data acquisition, balanced charging, power estimation and communication display etc. Each control unit through the CAN bus for data communications, in order to estimate the single battery and battery module voltage, total voltage, charge and discharge current and temperature data acquisition and measurement, energy (SOC). At the same time, a distributed battery management system has strong expansibility, can carry out specific cell diagnosis and safety performance of the battery protection function expansion. The overall structure of the battery management system, as shown in Figure 2. 


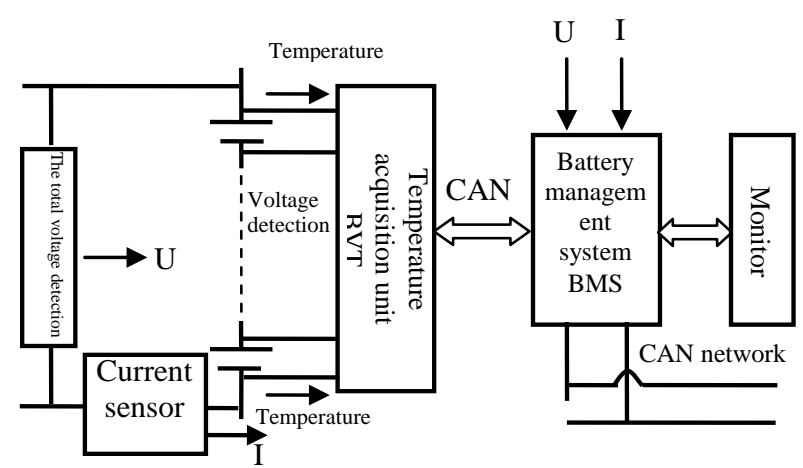

Figure 2. Schematic Diagram of Battery Management System Structure

The overall framework of the system analysis: battery management system (BMS) is the core of the control part of the whole system, the main function is data sampling control, digital signal preprocessing, Kalman filtering optimization algorithm realization, the PC serial communication, a battery protection circuít contro and other peripheral module management. First of all, the temperature acquisition unit (BVT) for real-time measurement of voltage, current, temperature and other parameters of the battery, to obtain the corresponding AD sampling data Then, the temperature acquisition unit (BVT) does preprocessing of these data, the batterymanagement system (BMS) implement SOC algorithm to get the current value of the remaining battery power. According to the SOC estimation value and other parameters information of battery, battery management system (BMS) for diagnosis of the state of the battery pack, if the battery over charge, over discharge and other abnormal phenomena, the battery management system (BMS) will immediately cut off the charging and discharging circuit. Finally, the battery management system (BMS) transmits battery test) data to monitor and the battery SOC estimation of measured data DC screenthrough the CAN communication.

\section{The Study of Remaining Battery Power (SOC) Estimation Method}

In the lithium iron phosphlate battery during use, the remaining battery power and is affected by many uncertain factors. How to use the battery measurable parameter data to achieve the accurate estimation of the remaining battery power is always the core issues and technical difficylties in urgent need of lithium iron phosphate battery management system to solve.

\subsection{The Definition of SOC}

Simply speaking, SOC refers to the storage battery power, the remaining power. It is the main parameter of battery status. Typically, SOC value is defined as the ratio of the remaining battery and battery capacity when discharging in a certain environment:

$$
S O C=\frac{Q_{c}}{Q_{n}}=1-\frac{Q_{i}}{Q_{n}}=1-\frac{\eta Q_{I}}{Q_{n}}
$$

In the formula: $Q_{n}$ is a nominal capacity; $Q_{c}$ is the remaining battery power refers to all of the electricity obtained the current state of charge of battery full discharged to the discharge cut-off voltage at the room temperature of $25 \mathrm{DEG} \mathrm{C}$ and $\mathrm{C} / 30$ rate; $Q_{i}$ as standard battery has electricity, is numerically equal to the difference between nominal capacity and standard residual quantity; $Q_{I}$ is the actual has electricity consumption, refers to the release of the power of the battery fully charged battery at the actual temperature and discharge rate. $\eta$ is the battery efficiency coefficient was used to quantify the impact of various factors on the battery SOC. 


\subsection{The Effect of SOC}

In the running environment of the power system, SOC is influenced by discharge rate, battery temperature, self-discharge rate, the number of recycling and many other factors, it is difficult to estimate SOC.

(1) discharge rate

When other factors under the same conditions, discharge capacity of the battery will decrease with the increase of the discharge rate. This is because the depth along the thickness direction of the electrode active substance inside the battery is limited, when the large current discharge, the higher discharge rate is, the more shallow depth is, the lower the utilization rate is, so the battery capacity is smaller, otherwise, the battery capacity is greater. Figure 3 is the constant current discharge characteristics curve at room temperature 25 degrees of lithium iron phosphate battery discharge rate in $0.25 \mathrm{C}$ dind $0.85 \mathrm{C}$.

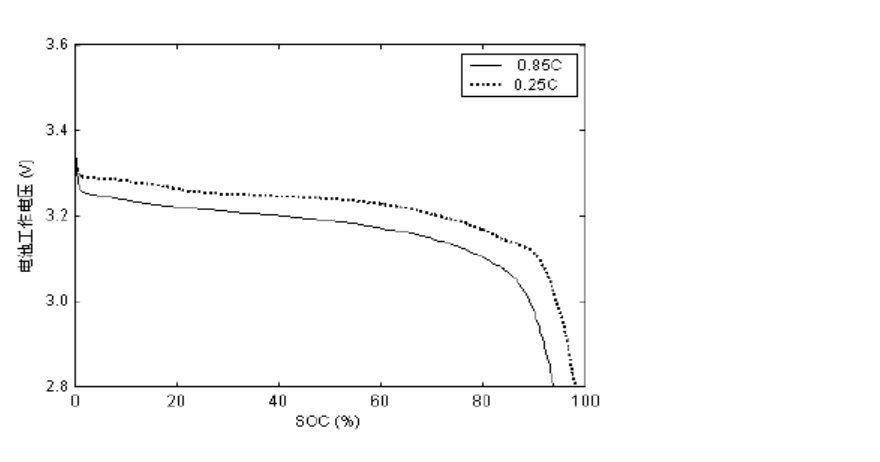

Figure 3. The Discharge Rate Characteristic Curve At Room Temperature

(2) The temperature of the battery

The utilization rate, of Iithium iron phosphate battery and active substances will increase with the rise of the temperature of the battery, which is mainly caused by the change of the temperature of the electrolyte performance. When the battery temperature rises, the electroyte viscosity decreases, the activity increases, lead to the enhancement of ion diffusion abihty, eventwally making active material utilization rate raised, the lithium iron phosphate battery actual available capacity increases. On the contrary, the battery temperature is decreased to reduce the rate of active material utilization, the actual available capacity s reduced, so the battery power is proportional to the actual availability and battery temperature. In actual use, the charge discharge temperature range of lithium iron phosphate battery: $0 \sim 45$ degrees.

(3) Cycle number

After a period of use of lithium iron phosphate battery, the total standard energy will change. At the beginning, the electricity will increase, and remained largely unchanged in the next period of time, then the battery will gradually reduce. For the lithium iron phosphate battery, the battery cycle life can be expressed with charge and discharge times of the total power can be put to nominal consumption $80 \%$.

(4) Self-discharge rate

Self-discharge also called charge retention, refers to a certain environmental conditions, Storage energy retention ability of battery under the open state. Under the action of the battery self-discharge, the SOC value decreases with the increase of the storage time. In general, said that since the percentage of discharge rate decreased with the capacity per unit time. Self-discharge rate $=\frac{\left(\mathrm{C}_{a}-C_{b}\right)}{C_{a} * T} * 100 \%$

In the formula: $\mathrm{C}_{a}$ is stored before the battery, $C_{b}$ is storage battery, $T$ is battery storage time. The self-discharge rate of lithium battery is related to relevant factors of 
recycle times, environmental temperature and storage time, which is calculated by the use of test methods are generally.

\subsection{Common Method of SOC Estimation}

Lithium iron phosphate battery in the DC power of discharge is a complex process of electrochemical reaction, discharge rate, environment temperature, the internal resistance of the battery, self-discharge rate, and other factors will value of battery SOC estimation influence, also these factors will change with the increase in the number of recycling, and a corresponding increase in the battery modeling and algorithm of SOC estimation difficult.

At present, domestic and foreign iron phosphate lithium battery SOC estimation methods mainly include discharge test, open circuit voltage method ah measurement method, the analysis of these methods are briefly introduced.

(1) Discharge test method

The discharge test is experimental method using constant current battery voltage continuous discharge until the battery reaches the discharge voltage, the remaining battery power is equal to the current value of product and time of discharge. The discharge test method is often used in the laboratory, is the most reliable method of SOC estimation, and is applicable to all types of batteries, but it also has defects the thellowing two aspects: first, the need to spend a lot of time measurement. Only when the discharge end of the trial, before the moment of the SOC valie can be calculated, unable to do the SOC estimation in real time; the second, before the battery to was forced to stop, and go to the constant current discharge state.

(2) Open-circuit Voltage

The open circuit voltage of the cell in the numerical and electric potential of battery is very close. Lithium iron phosphate batterpelectromotive force is about the internal electrolyte density function and electrolyte density will increase with the number of battery discharge and decreased in propotion to the, so the open circuit voltage method is according to battery under certain donditions of open circuit voltage and SOC values into a mathematical reationship between the proportion of the principle to estimate SOC. At the end of discharge, the open circuit voltage good estimates of the effect of SOC. But the open circuit voltage methed itself also has some shortcomings, such as open circuit voltage measurement time In order to overcome the effect of self-recovery. The battery needs long time standing to reach steady state voltage, generally the static process takes several hours to ten hours, which resulted in the waste of time; in addition, how to correctly judge the battery is reached steady state is left more than power estimation difficult. When the battery is in the middle of the discharge platform, the numerical relationship between open circuit voltage and SOC is not very obvious, SOC leads to larger estimation error.

(3) Measurement on time

Measurement on time is to estimate the battery SOC by calculating the cumulative power in the battery charge or discharge time, and according temperature, charge and discharge rate to compensate to the estimated SOC. It is currently the most widely used, and the simplest SOC estimation method has been successfully applied to the electronic consumer goods quantity estimation. If the specified initial state of charge and discharge is SOC0, then the current state of the SOC value can be calculated by the formula (3).

$$
S O C=S O C_{0}-\frac{1}{Q_{n}} \int_{0}^{t} \eta i d t
$$

In the formula: $Q_{n}$ is the nominal capacity; $i$ is battery current, when discharge is positive, while charging is negative; $\eta$ is the cell efficiency coefficient, including temperature influence coefficient $\eta_{T}$ and charging-discharging coefficient $\eta_{i}$, and $\eta_{i}$ can 
obtained by Peukert equation. In the use of on time measurement method should pay attention to three aspects: method itself cannot provide battery initial value $\mathrm{SOC}_{0}$; not accurate current measurement will increase SOC estimation error, after long time accumulation, the error will become bigger and bigger; when estimating SOC the battery efficiency coefficient $\eta$ must be taken into account. Although the accuracy of current measurement problems can be solved by using high performance current sensor, but it will make the system cost increase. At the same time, to solve the problem of battery efficiency coefficient $\eta$ must establish temperature coefficient $\eta_{T}$ and experience formula of charge-discharge rate coefficient $\eta_{i}$ by a large number of experimental data.

(4) Internal resistance measurement

Method of internal resistance measurement is refers to the method of measuring battery internal resistance at different frequency AC excitation of the battery, and calculating SOC value by using the battery remaining power and static model AC resistance. Late in the discharge of the battery, internal resistance measurement method has high accuracy of estimation and the battery adaptability, generally under the on time measurement method used in combination.

\section{Conclusions}

This paper introduces the application of lithium iron phosphate battery in DC system in substation, application analysis and study on the battery management system in lithium iron phosphate battery group and SOC algorithm. Battery management system is in charge of the entire battery operation. It needs to monitor the operation of the battery, aceurate estimation of residual capacity of battery, adjust the difference between the battery cell, provide a variety of battery fault protection and alarm information. Battery management system and DC control system communicate through CAN, can effectively ensure the safe and reliable work of lithium iron phosphate, battery and the DC system.

\section{References}

[1] H.-M. Xu and Q.-S. Wâng, The Use and Maintenance of DC Power Supply Equipment of Substation", Beijing: China Electric Power Press, (2007).

[2] Y.-H. Zhou, J. Chen and X.-F. Jiao, "Progress of LiFePO Cathode Material for Lithium Ion Batteries", Power Supply Technology, vol. 33, no. 8, (2009), pp. 649-651.

[3] J. Li, Y. Zhang and J.L. Li, "Iron Phosphate Potassium Battery Feasibility Analysis in Application of Substation Systen", East China Electric Power, vol. 37, no. 10, (2009), pp. 1693-1695.

[4] X.-M. Xlang B.-M. Zhou and S.-M. Xu, "The Investigation of the Storage Battery Capacity On-line Monitorng\%, Power Supply Technology, vol. 33, no. 3, (2009), pp. 213-216.

[5] G.-S. Hu, C.-C. Chen, X.-M. Zhang and Y.-T. Pan, "To Improve The Life of Lead-acid Battery By Battery Equalization", Power Supply Technology, vol. 30, no. 7, (2006), pp. 576-579.

[6] H. Wang, G.H. Zhang and Z.-Q. Liang, "Network Management and Maintenance of DC Power Supply System", Power System Technology, vol. 4, no. 2, (2001), pp. 185-189. 
International Journal of Control and Automation

Vol. 9, No. 8 (2016)

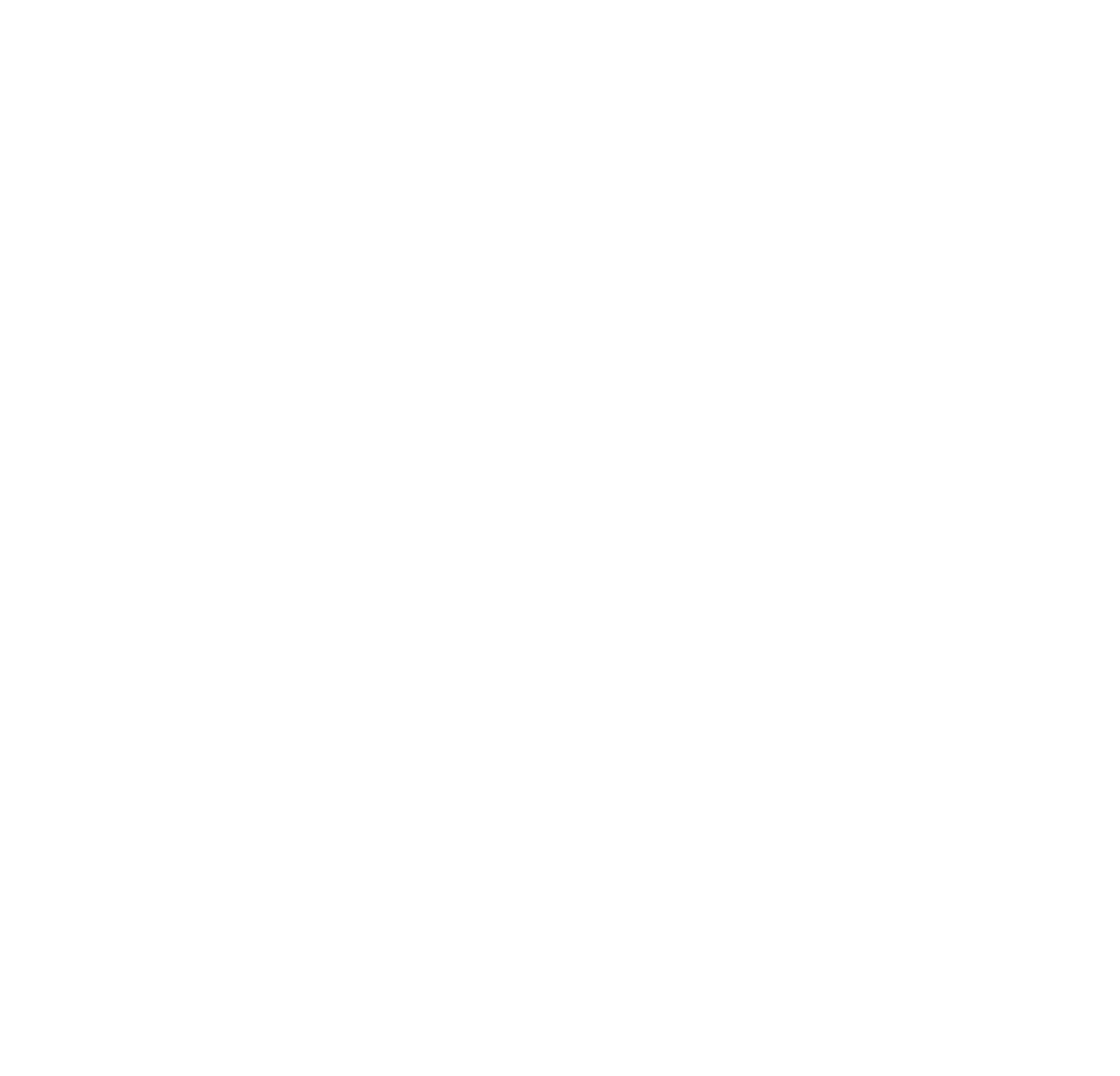

